\title{
Flight of Ideas
}

National Cancer Institute

\section{Source}

National Cancer Institute. Flight of Ideas. NCI Thesaurus. Code C117266.

Rapid succession of thoughts pertaining to different subjects that are still connected. 